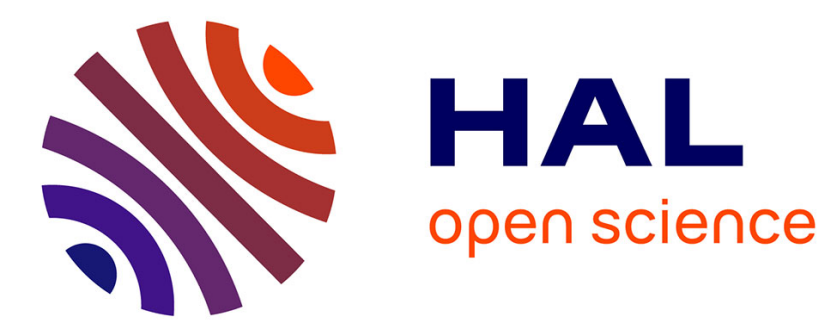

\title{
SUPERCONDUCTIVITY IN METALLIC GLASSES
}

W. Johnson

\section{To cite this version:}

W. Johnson. SUPERCONDUCTIVITY IN METALLIC GLASSES. Journal de Physique Colloques, 1980, 41 (C8), pp.C8-731-C8-741. 10.1051/jphyscol:19808183 . jpa-00220286

\section{HAL Id: jpa-00220286 https://hal.science/jpa-00220286}

Submitted on 1 Jan 1980

HAL is a multi-disciplinary open access archive for the deposit and dissemination of scientific research documents, whether they are published or not. The documents may come from teaching and research institutions in France or abroad, or from public or private research centers.
L'archive ouverte pluridisciplinaire HAL, est destinée au dépôt et à la diffusion de documents scientifiques de niveau recherche, publiés ou non, émanant des établissements d'enseignement et de recherche français ou étrangers, des laboratoires publics ou privés. 


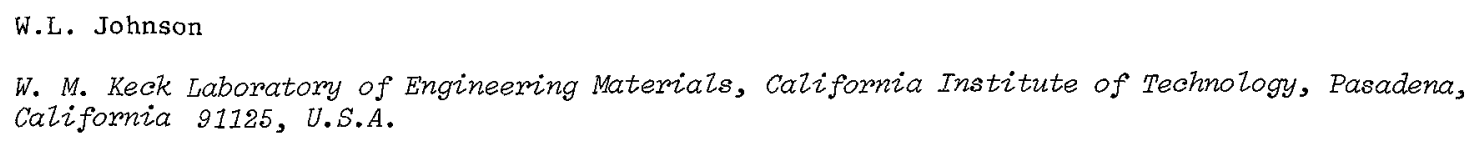

\section{INTRODUCTION}

The earliest studies of amorphous superconductors were carried out twenty-five years ago on thin films of simple metals prepared by vapor deposition on a cryogenic substrate ${ }^{(1,2)}$. Bergmann ${ }^{(3)}$, and the author $(4)$ have surveyed some of the properties of such materials in recent reviews. In the past ten years, studies of amorphous superconductors have been extended to transition metals $(5,6)$, and most recently to metallic glasses $(4,6,7)$. The latter materials are prepared by rapid quenching of liquid metallic alloys using techniques originaliy developed by Duwez ${ }^{(8)}$.

It would be both difficult and repetitive to completely review this field in the limited space available here. The interested reader is referred to the above mentioned articles for a more complete survey. In the present article, attention will be focused on recent developments. The main results of earlier work will be summarized where convenient.

\section{ELECTRONIC STRUCTURE AND SUPERCONDUCTIVITY}

McMillan ${ }^{(9)}$ has given a solution to the strongcoupling equations of Eliashberg which he obtained using numerical techniques together with the measured phonon spectrum of niobium. Using his solution, the superconducting transition temperature is given by

$$
T_{C}=\left[{ }_{0} / 1.45\right] \exp -\left[\frac{1.04(1+\lambda)}{\lambda-\mu^{*}(1+0.62 \lambda)}\right]
$$

In this expression, $\theta_{D}$ is the Debye temperature (or suitably averaged phonon frequency), $\mu^{*}$ the effective Coulomb coupling constant, and $\lambda$ the dimensionless electron phonon coupling constant. IICMillan expresses $\lambda$ in terms of other microscopic parameters as

$$
\lambda=\frac{D(0)<I^{2}>}{M<\omega^{2}>}
$$

$$
\begin{aligned}
& \text { where } D(0)=\text { electron density of states at } \\
& \text { the Fermi Tevel } \\
& \left\langle I^{2}\right\rangle=\text { average squared electron-ion } \\
& \text { matrix element } \\
& M=\text { ionic mass } \\
& \left\langle\omega^{2}\right\rangle=\text { mean square phonon frequency } \\
& \text { (defined by McMilian) }
\end{aligned}
$$

Although the phonon spectrum and Eliashberg function $\alpha^{2}(\omega) F(\omega)$ of amorphous metals may differ from that used by licmillan to obtain eqn. (1), one would still expect that the corresponding expression for $\mathrm{T}_{c}$ in amorphous alloys will have a similar form $(4,10)$. Therefore, eqns. (1) and (2) still provide a convenient framework in which to anatyze the systematics of superconductivity in amorphous metals.

\section{A. Simple Metals}

The occurrence of superconductivity in amorphous simple metals can be understood by using an extension of the nearly free electron model.

Ziman has applied this model to liquid metals (11) Bergmann ${ }^{(3)}$ has given an extensive account of the experimental evidence which supports such a picture in the case of superconducting amorphous simple metals. In particular, the model correctly predicts the $H a 11$ coefficient ${ }^{(12)} R_{H}$, electrical resistivity $\rho(T)$, and temperature dependence of $\rho(T)$ for the majority of simple metals $(13)$. Rainer and Bergmann ${ }^{(14)}$ have also discussed the calculation of $\left\langle I^{2}\right\rangle$ and $\left\langle\omega^{2}\right\rangle$. They argue that disorder results in an enhancement of low frequency contributions to electron-phonon scattering and consequently to enhanced values of $\lambda$. They conclude that amorphous metals should tend to be strong-coupling $(\lambda>1)$ superconductors. 
Experimental evidence obtained from superconductive tunneling indeed supports this conclusion $(3,15,16)$. The author ${ }^{(4)}$ has attempted to explain the systematics of superconductivity in simple amorphous metals by assuming that the strong disorder leads to a nearly spherical Fermi surface. A simple jellium model was used to determine the variation of $\lambda$ with a few simple parameters such as valence $Z$, atomic volume $v_{a}$, and ionic mass $M$. In the jellium model, the ionic plasma frequency $\Omega_{p}=\left(\frac{4 \pi \mathrm{e}^{2}}{M}\right)^{\frac{3}{2}}$ (where $N=$ density of ions) is a natural scale for phonon frequencies. A plot of the dimensionless ratio. $\left[k_{B} T_{C} / \hbar \Omega_{p}\right]$ for amorphous simple metals as a function of average valence $Z$ is shown in Fig. 1 and suggests remarkably systematic relationship. Roughly speaking, this ratio is a measure of the exponential factor on the right hand side of eqn. (1), and thus a measure of $\lambda$. Using $Z, v_{a}$, and $H$ as input parameters in the jellium model, together with the Heine-Abarenkov pseudopotential form factors for simple metals, a simple model calculation of $\lambda$ was carried out ${ }^{(4)}$. It was found that $\lambda$ is determined mainty by valence. These results are illustrated in Fig. 2 .

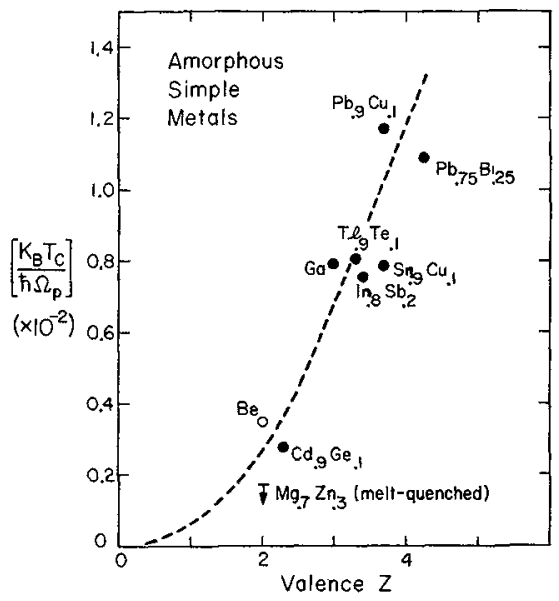

Fig. 1. Variation with valence of the superconducting transition temperature of simple amorphous metals. The values of $T_{c}$ are normalized to the characteristic bare phonon temperature $\left[h \Omega_{\mathrm{p}} / \mathrm{K}_{\mathrm{B}}\right]$ of each material.
In crystalline metals, the influence of long range order on electronic band structure results in large deviations of the electronic density of states $D(\varepsilon)$ from the free electron model. These deviations in turn influence electron-phonon scattering, and electron screening. The absence of long range order in amorphous metals tends to eliminate these band structure effects and leads to a free electron-like Fermi surface. As a result, the jellium model gives a much better description of amorphous as compared to crystalline simple metals which are not well described by the jellium model ${ }^{(4)}$. The microscopic origin and systematics of superconductivity are correspondingly easier to understand in amorphous metals.

\section{B. Transition Metals}

The superconducting properties of amorphous transition metals are governed mainly by the partially occupied d-states. In contrast with the case of simple metals, one cannot use the free electron approach to understand these materials. The d-electrons are perhaps best described as being tightly bound. Friede ${ }^{(18)}$, Cyrot-Lackmann ${ }^{(19)}$, and others have discussed the tight-binding approximation TBA as it applied to liquid transition metals. Recently, Varma and Dynes ${ }^{(20)}$ have analyzed superconductivity in crystalline transition metals using an extension of the TBA method to include the nonorthogonality of atomic d-orbitals centered on neighboring atoms. They find that the d-band contribution $D_{d}\left(\varepsilon_{F}\right)$ to $D\left(\varepsilon_{F}\right)$ is the most important microscopic parameter which determines $\lambda$. Their argument shows that the ratio $\left[\left\langle I^{2}\right\rangle /\left\langle\omega^{2}\right\rangle\right]$ in eqn. (2) should be roughly constant within a given d-band (i.e. the $4 d$ or $5 d$ band).

Applying the Varma-Dynes analys is to the case of amorphous transition metals requires information regarding the behavior of $D_{d}(\varepsilon)$. Severai experimental techniques have been used to obtain such information. These include measurements of magnetic susceptibility, low temperature specific heat, $x$-ray and ultraviolet photoemission spectra, and also indirect deduction of $D\left(\varepsilon_{F}\right)$ from upper critical field, $\mathrm{H}_{\mathrm{c} 2}(T)$, measurements. Magnetic susceptibility measurements on metallic glasses of the series $\left(\mathrm{MO}_{1-x} \mathrm{Ru} \mathrm{x}_{x}\right)_{80} \mathrm{P}_{20}$ have been reported ${ }^{(21)}$. The temperature independent contribution to the susceptibility, $x_{0}$, was found to be large and to decrease with $x$ as shown in Fig. 3. If one 


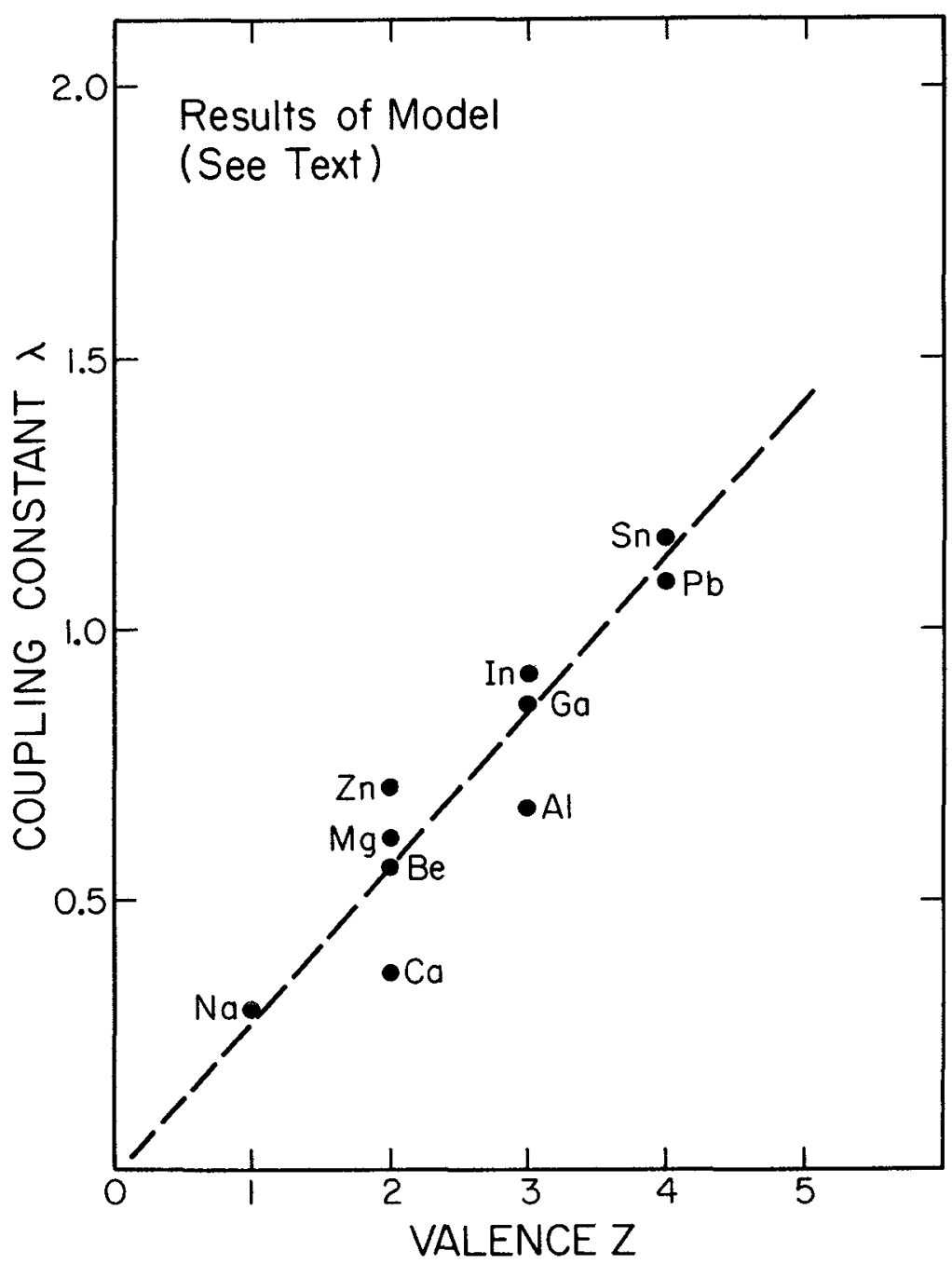

Fig. 2. Values of the electronphonon coupling constant $\lambda$ calcuTated using a simple jellium model with $Z, M$, and $v_{a}$ as input parameters. See ref. 4 for details.

assumes that the Pauli paramagnetic contribution associated with $D\left(\varepsilon_{F}\right)$ dominates $\chi_{0}$, then this can be interpreted to indicate a smooth decrease of $D\left(\varepsilon_{F}\right)$ with increasing $x$ as shown in the figure. Also shown in the figure is the variation of $T_{C}$ with $x$ for these metallic giasses and the variation of $T_{C}$ with $x$ for amorphous $\mathrm{Mo}_{1-x} R u_{x}$ thin films prepared by cryoquenching by. Collver and Hammond ${ }^{(4)}$. These results suggest a direct relationship between $D(\varepsilon)$ and $T_{C}$. Recent low temperature specific heat measurements ${ }^{(4)}$ on the same series of metallic glasses have confirmed the decrease of $D(\varepsilon)$ with $x$. The absolute values of $D(\varepsilon)$. obtained from the coefficient of the linear contribution to $c_{p}$ are in remarkably good agreement with $D(\varepsilon)$ values estimated from $x_{0}{ }^{(4)}$. Schroeder et aT ${ }^{(22)}$ and Amamou and the author ${ }^{(23)}$ have used XPS and UPS measurements to gain insight into the variation of $D(\varepsilon)$ for $\varepsilon<\varepsilon_{F}$. Typical results are shown in Fig. 4 for the alloy $\left(\mathrm{H}_{0} \mathrm{o}_{0.6} \mathrm{Ru}_{0.4}\right)_{80} \mathrm{~B}_{20}$. The data suggest that a general loss of structure in $D(\varepsilon)$ occurs on going from the crystalline to the amorphous state. It also suggests that $D(\varepsilon)$ is characterized by a broad maximum with the peak occurring somewhat below the Fermi level of the alloy. In other words, $D_{d}(\varepsilon)$ appears to vary rather smoothly with d-band occupation exhibiting a maximum for a roughly half filled $d$-shell. The variation of $T_{c}$ observed by Collver and Hammond ${ }^{(4)}$ for cryoquenched films of transition metals and alloys of neighboring metals of the $4 \mathrm{~d}$ series is shown in Fig. 5. The variation of $T_{c}$ through a similar broad maximum is consistent with the Varma-Dynes analysis if the density of 


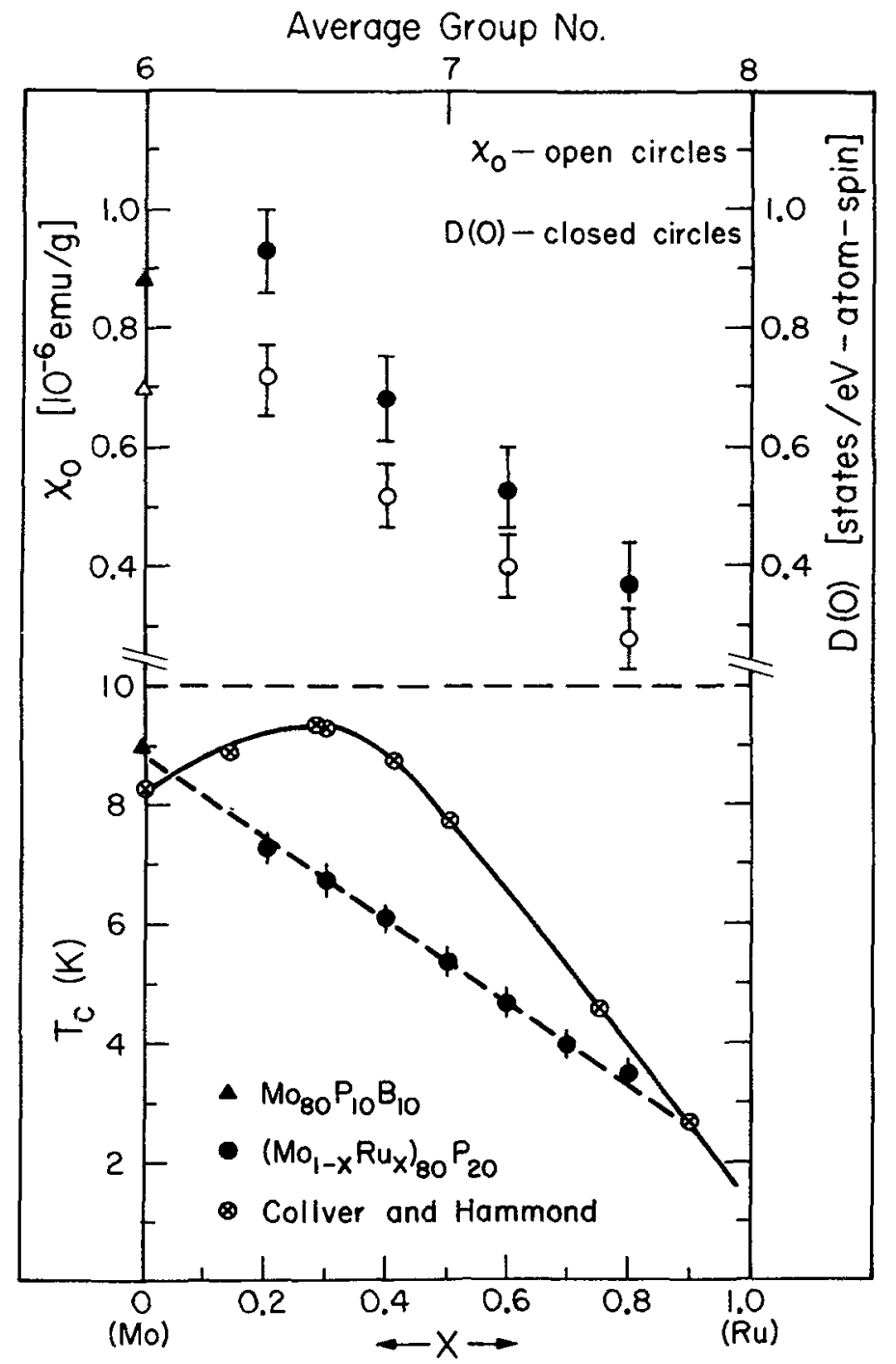

Fig. 3. (top) The temperature independent contribution $x_{0}$ to the magnetic susceptibility and corresponding density of states $D(0)=2 \mu_{B}^{2} x_{0}$ estimated by assuming $x_{0}$ is dominated by Pauli paramagnetism.

(bottom) The variation of $T_{c}$ for the same alloys along with that observed by Collver and Hammond for $\mathrm{Mo}_{1-x} \mathrm{Ru}_{x}$ thin films.

states $D_{d}(\varepsilon)$ has the above characteristics. In contrast, the Matthias curve for crystalline transition metals is shown in the same figure. In the crystalline case, a rapid variation of $D_{d}\left(\varepsilon_{F}\right)$ with d-band occupation is predicted by band structure calculations $(24,25)$ and confirmed by specific heat measurements $(9,20,26)$. Those crystalline metals and alloys for which $\varepsilon_{F}$ falls near a sharp maximum in $D(\varepsilon)$ have high $T_{C}$ values as expected in the Varma-Dynes model. The crystalline to amorphous transition washes out the $d$-band structure and thus leads to a smoother variation of both $D_{d}(\varepsilon)$ and consequently, $T_{c}(4)$.

\section{Effects of Disorder on Superconductivity}

In amorphous metals, essentially every atom can act as a scattering center for conduction electrons. This is due to the highly disordered atomic arrangement. In the case of simple metals, the atomic potential is comparatively weak. The Ziman theory of liquid metals $(11)$ is premised on the assumption of weak scattering and as such neglects multiple scattering effects. Using the simplest solution to the Boltzmann equation, one can estimate the electronic mean free path for amorphous simple metals directly from measurements of $\rho(T)$. At cryogenic temperatures, $\rho(T+0)$ ranges from 25-100 $\mu \Omega \mathrm{cm}$ for typical simple metals. From

$$
p=\frac{m v_{F}}{n e^{2} l_{e}}
$$

and free electron values for the Fermi velocity, 


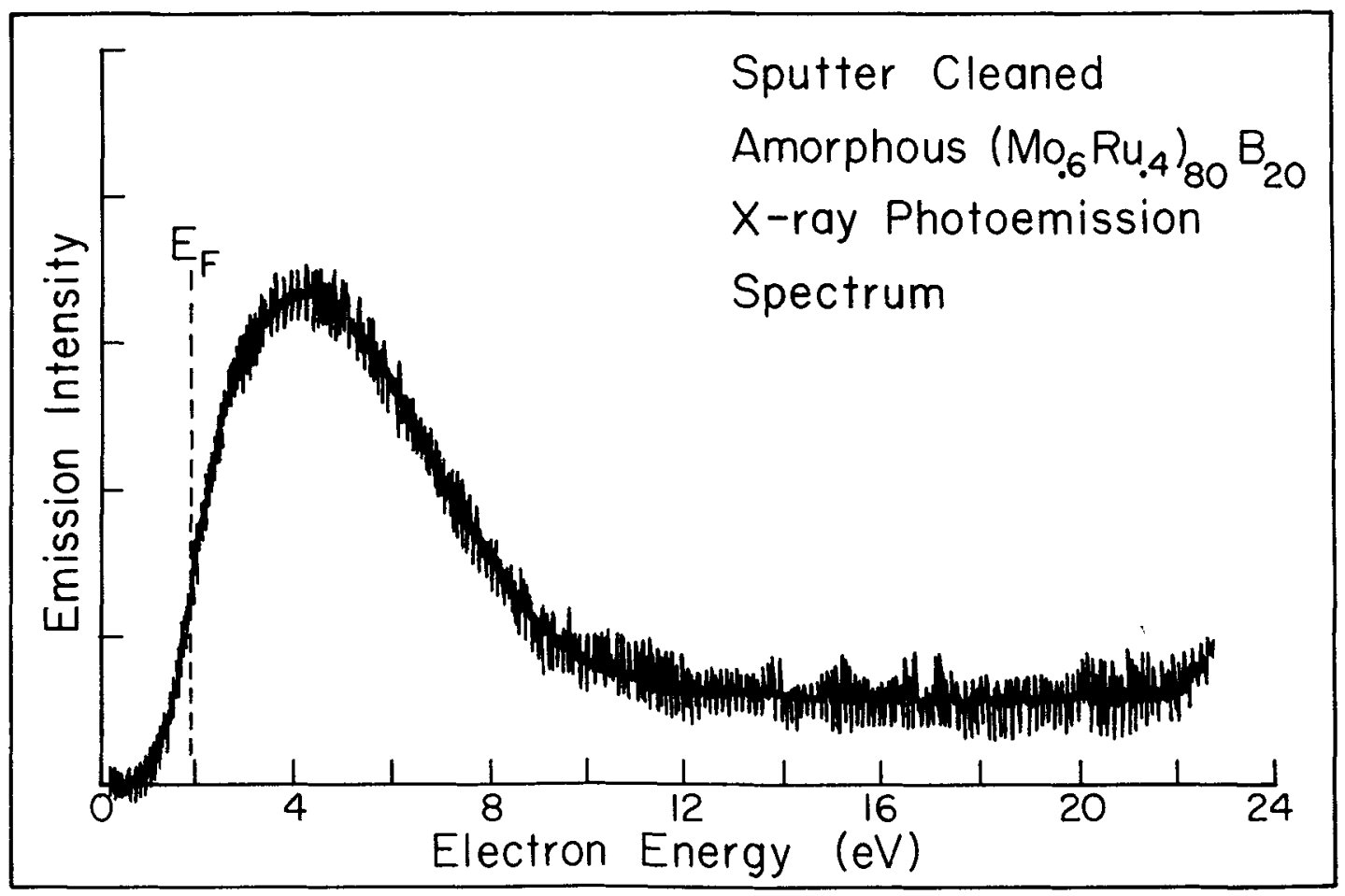

Fig. 4. The $x$-ray photoemission spectrum of $\left(M_{0} 0_{0}{ }^{R u_{0.4}}\right)_{80} B_{20}$ shows the width and shape of the $d$-band. UPS spectra are given in ref. 22 .

$v_{F}$, one obtains an electron mean free path $\ell_{e}$ which ranges from several times the interatomic distance $\mathrm{d}$ up to roughly $10 \mathrm{~d}$. The Ziman model is expected to break down when $\ell_{e} \approx d$. Mott has termed this the electron diffusion $(27)$ or strong scattering regime and predicts that this regime is approached when $\rho \geq 300 \mu \Omega \mathrm{cm}$. Resistivities of this order are in fact observed in amorphous transition metals where eqn. (3) gives values of $\ell_{e} \approx d$. It should be noted that still larger values of $\rho$ (i.e.

$\rho \geq 3000 \mu \Omega \mathrm{cm})$ are expected to result in Anderson localization of electronic states ${ }^{(28)}$.

The small value of $\ell_{e}$ typically found for amorphous metals $(27,28)$ has important consequences for superconductivity. The purity parameter $\lambda_{p}=\left(\xi_{o} / \ell_{e}\right)$ where $\xi_{0}$ is the zero temperature coherence length in the clean limit $\left(\xi_{0}=\frac{\hbar v_{F}}{\pi \Delta}(29)\right.$ is exceptionally large, $\lambda_{p} \sim 10-100$, for amorphous metals. Thus, amorphous metals are a case of superconductivity in the extreme dirty limit ${ }^{(29)}$. In this limit the coherence length becomes

$$
\xi_{d}(t)=0.85\left[\xi_{0}(T) \ell_{e}\right]^{\frac{1}{2} t^{-\frac{1}{2}}}
$$

where $t$ is the reduced tempertature $t=\left(\frac{T-T_{C}}{T_{c}}\right)$ ) while the penetration depth is given by $(29)$

$$
\lambda_{d}(t)=0.615 \lambda_{L}(0) \lambda_{p}^{\frac{1}{2}} t^{-\frac{1}{2}}
$$

where $\lambda_{L}(0)$ is the London penetration depth. For amorphous metals $\xi_{d}(0)$ is in the range $30-100 \AA$ while $\lambda_{d}(0)$ is of the order of $1 \mu \mathrm{m}$. The GinzburgLandau parameter $k=(\lambda / \xi)$ is of order $10-100^{(30,31)}$.

As a consequence of the above facts, amorphous superconductors have a large upper critical field $H_{c 2}(T)^{(30,31)}$, small lower critical field $\mathrm{H}_{\mathrm{Cl}}(\mathrm{T})^{(30)}$, and exhibit large critical fluctuations ${ }^{(32)}$. The upper critical field behavior is particularly interesting and has recently been studied over an extended range of $t$ for amorphous transition metal alloys by Tenhover, Tsuei, and the author (31). Examples of these data are shown 


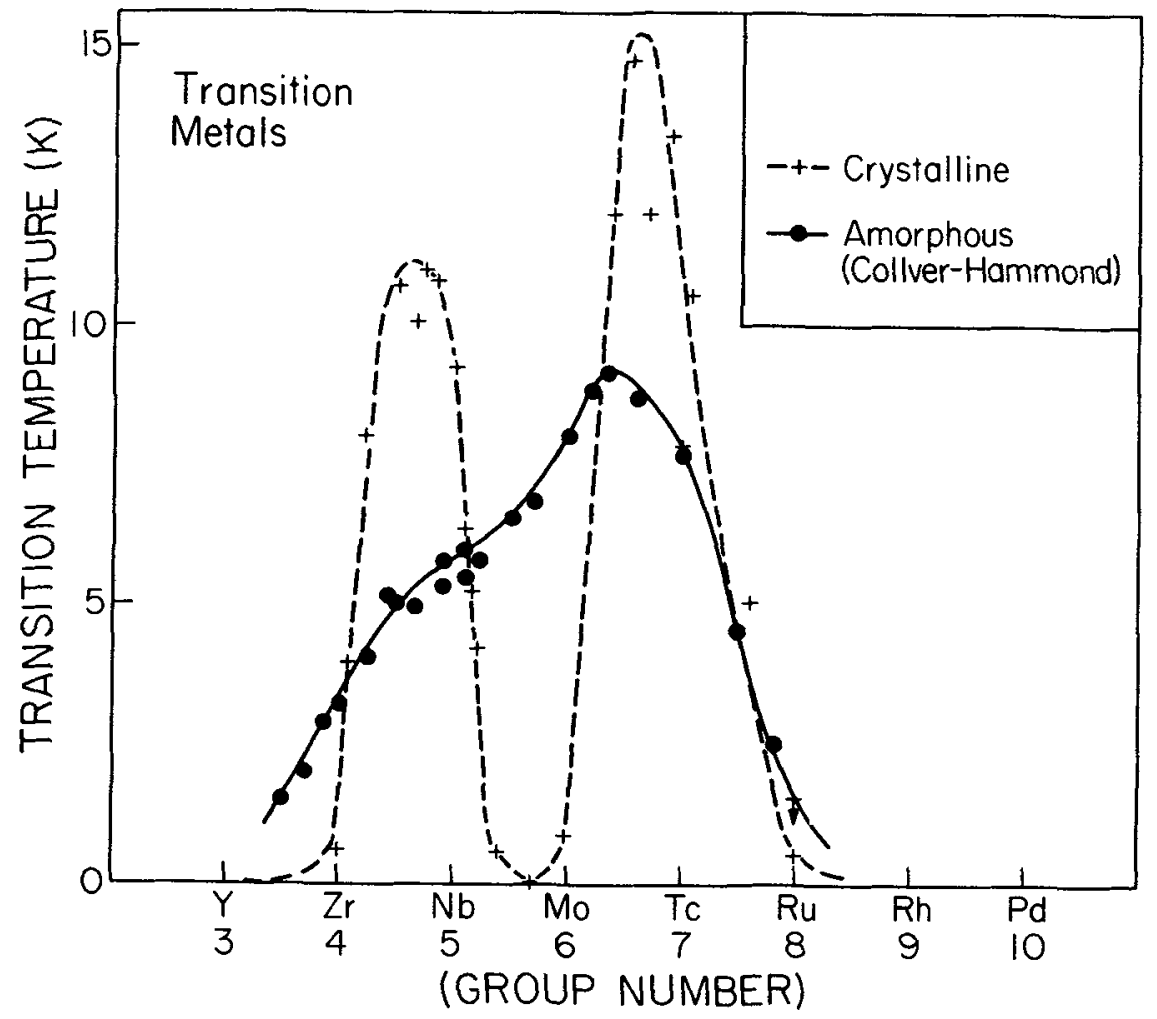

Fig. 5. The variation of $T_{c}$ with average group number for thin amorphous films of $4 d$ transition metals and alloys. For comparison, the variation of $\mathrm{T}_{\mathrm{C}}$ in corresponding crystalline metals and alloys is shown.

in Fig. 6. The theoretical calculation of $\mathrm{H}_{\mathrm{c} 2}(\mathrm{~T})$ in the dirty limit has been carried out by several authors $(33,34,35)$. Corrections to the basic theory for spin orbit scattering $(35)$, paramagnetic limiting ${ }^{(36)}$, strong-coupling ${ }^{(37)}$, an anisotropy effects ${ }^{(38)}$ have been successful in explaining most data on known superconductors. Exceptions to this included recent results on ultra thin films ${ }^{(39)}$, and the data in Fig. 6. The figure shows the theoretical curves for $\hbar(t)=\left(H_{c 2}(T) / H_{c 2}^{L}(0)\right)$ where $H_{\mathrm{c} 2} \mathrm{~L}(0)$ is obtained by 7 inear extrapolation. By letting the spin-orbit coupling parameter $\lambda_{\text {so }} \rightarrow \infty$, one obtains the highest appropriate theoretical prediction for $\hbar(t)$. The theory is clearly inadequate to explain the persistent linearity of $\hbar(t)$ at low $t$. Combining the data of Fig. 6 with all other available data on amorphous reveals that the discrepancy with theory systematically increases with increasing normal state resistivity $\rho^{(31)}$. Values of $\rho$ of the order of $200 \mu \Omega \mathrm{cm}$ are observed for the alloys in Fig. 6 . On this basis, it is suggested that this behavior is related to strongelectron scattering effects and the tendency toward electron localization. Such effects would also be expected for the case of ultrathin films (39).

The topological and chemical disorder in amorphous transition metal alloys should result in large fluctuations in the atomic environment. In the TBA model of the d-band, one would expect large fluctuations in the two center overlap integral (17)

$$
J=\int \Phi_{d}^{*}\left(\vec{r}-\vec{r}_{1}\right) V_{a}\left(\vec{r}-\vec{r}_{1}\right) \Phi_{d}\left(\vec{r}-\vec{r}_{2}\right) d^{3} \vec{r}
$$

where $\vec{r}_{1}$ and $\vec{r}_{2}$ are the position vectors of neighboring atoms, $v_{a}$ the atomic potential, and $\Phi_{d}$ an atomic d-orbital. Roughly $J$ depends on $|\vec{l}|=\left|\vec{r}_{2}-\vec{r}_{1}\right|=\ell$ as

$$
J(\ell)=J_{0} e^{-\ell q_{0}}
$$




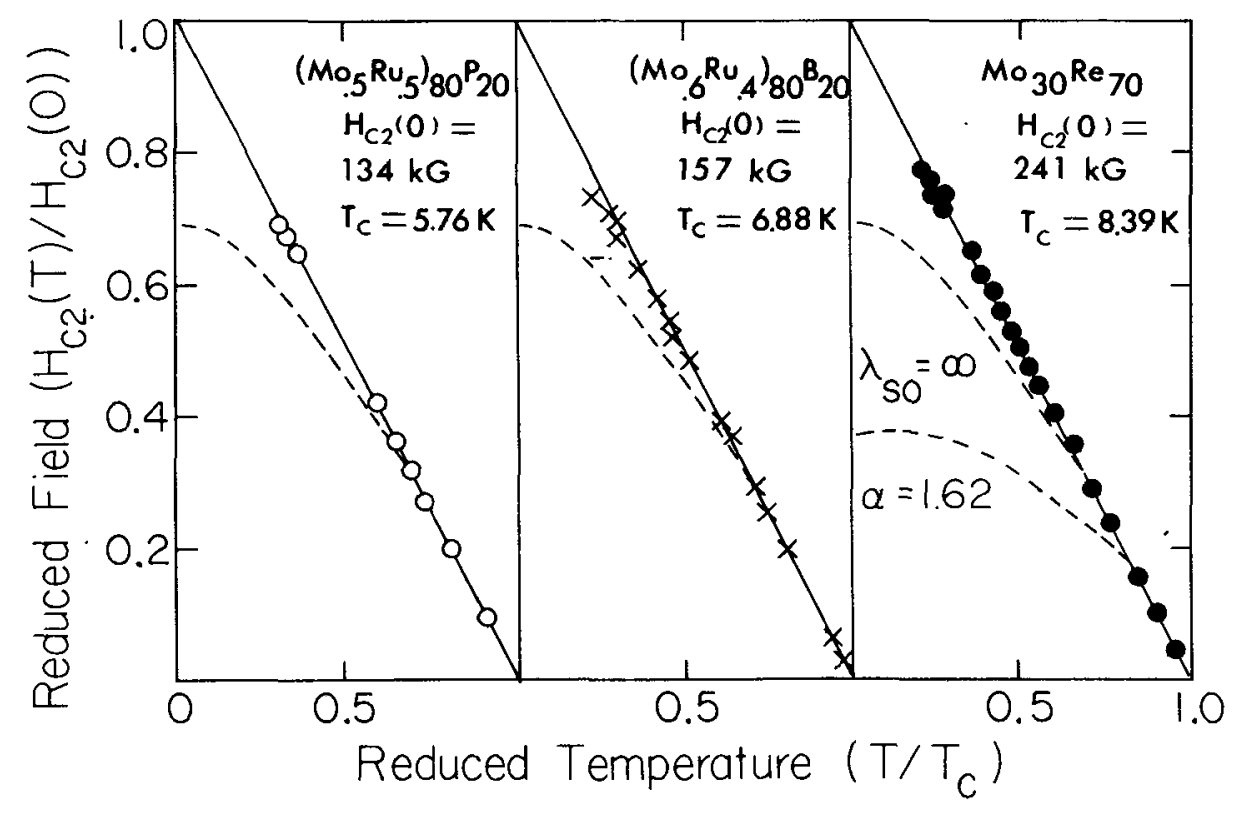

Fig. 6. The reduced upper critical field $h(t)=H_{c 2}(T) / H_{c 2}{ }_{L}(0)$ vs. $t$ for several transition-metal-base metallic glasses. Dashed lines are theoretical curves.

where $q_{0}$ is the slater coefficient for decay of the d-orbital. The d-band width is of order

$$
W_{d} \approx 2 z\langle\mathrm{~J}\rangle\langle\cos \theta\rangle
$$

where $z$ is the first neighbor coordination number. From the atomic pair correlation function of metallic glasses ${ }^{(4 l)}$ (e.g. $\left(W_{\left.0.5^{R u_{0.5}}\right)}\right)_{0} \mathrm{~B}_{20}$ we estimate the mean value of $\ell$ to be $\bar{l} \approx 3 \AA$ while fluctuations in $\ell$ are of order $\Delta_{\ell} \approx 1.0 \AA$. Taking $\mathrm{J}(\bar{l}) \approx 1 \mathrm{eV}$, and $q_{0} \approx 1 \AA^{-1}(40)$ gives $J_{0} \sim 10-30 \mathrm{eV}$. Thus the relative fluctuations in $J(\ell)$ are typically

$$
\left(\frac{\Delta_{\mathrm{J}}}{W_{\mathrm{D}}}\right) \approx\left(\frac{\Delta_{\ell}}{W_{\mathrm{D}}}\right)\left|\frac{\partial \mathrm{J}(\ell)}{\partial \ell}\right|_{\ell=\bar{l}}=\frac{q_{0} \Delta_{\ell}}{\mathrm{z}<\cos \theta>}
$$

The factor $<\cos \theta>$ accounts for the directional properties of the d-orbitals and for strongly directional $d$ orbitals we could take $z<\cos \theta>\sim 2$. Thus we estimate

$$
\left(\Delta_{\mathrm{J}} / W_{\mathrm{d}}\right) \sim \frac{1}{2} \approx \frac{1}{2}
$$

We see that topological disorder alone can produce substantial fluctuations in local environment.
Chemical disorder (e.g. fluctuations in Mo-Mo, Mo-Ru, and Ru-Ru coordination) will produce additional diagonal disorder. One might expect to approach the Anderson localization criteria for the d-band of a transition metal glass.

The above discussion ignores $s-d(p-d)$ hybridization which can be parametrized by a parameter $\mathrm{J}_{s d}$. If $\mathrm{J}_{\mathrm{sd}} \rightarrow 0$, then the $d$-electrons would be decoupled from the $s, p-e l e c t r o n s$ and could exhibit localization for sufficiently high disorder. For finite $J_{s d}$, the localization would tend to be suppressed by the greater spatial extent of $s, p$ oribtals. This interesting problem has important consequences for superconductivity since the d-electron time and spatial correlation effects play an important role in the microscopic theory of the inhomogeneous superconducting state.

\section{ATOMIC SCALE STRUCTURE AND MORPHOLOGY OF AMORPHOUS SUPERCONDUCTORS}

The simplified picture of the d-band density of states $D_{d}(\varepsilon)$ described in section II $B$. ignores the influence of metalloid elements (e.g. B, P, etc.) on electronic properties. Recent studies $(42)$ suggest the metalloid elements play a significant 


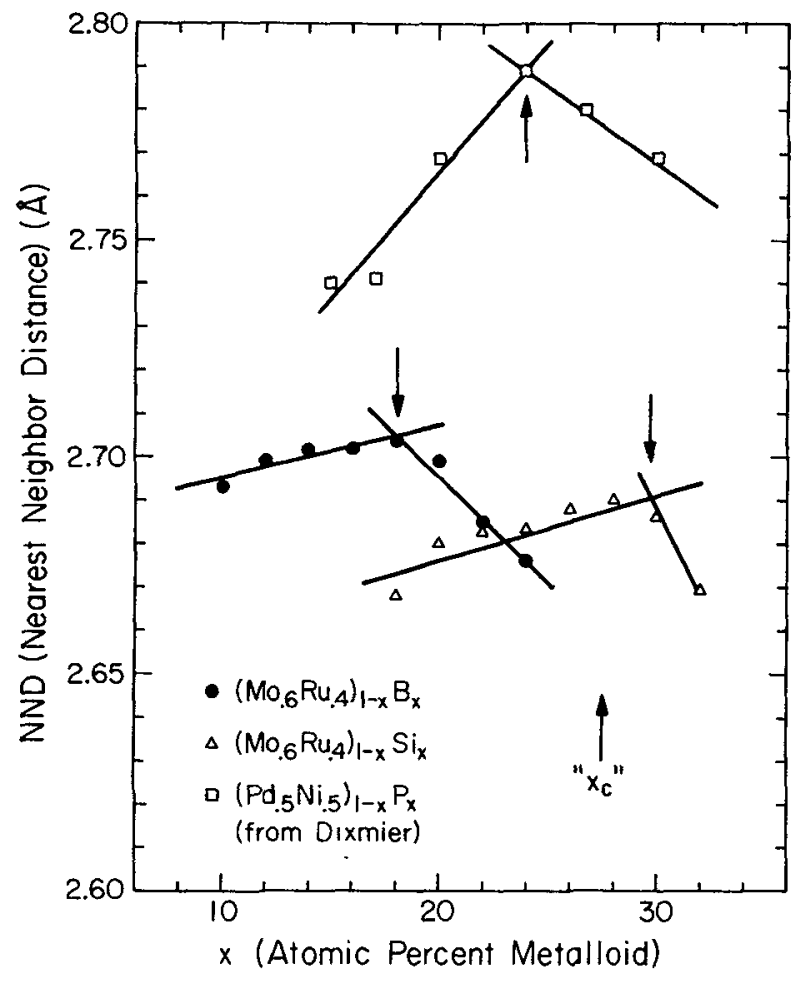

Fig. 7. Hearest neighbor distance $d$ vs. composition for ( $\left.\mathrm{Ho}_{0.6^{\mathrm{Ru}}} \mathrm{R}_{0.4}\right)_{1-\mathrm{x}} \mathrm{B}_{x},\left(\mathrm{Ho}_{0.6}{ }^{\mathrm{Ru}} 0.4\right)_{7-x} \mathrm{Si}_{x}$, and $\left(\mathrm{Pd}_{0.5} \mathrm{Ni}_{0.5}\right)_{1-\mathrm{x}^{\mathrm{P}} \mathrm{x}}$ metallic glasses. Distances were determined from the Debye formula. (see ref. 42)

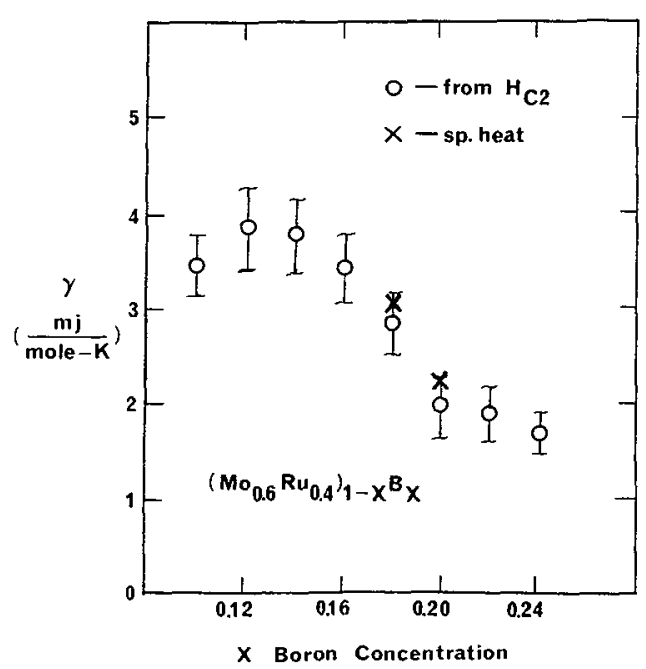

Fig. 9. Linear coefficient for low temperature specific heat vs. composition for $\left(\mathrm{flo}_{0.6}{ }^{\mathrm{Ru}} 0.4\right)_{7-x^{\mathrm{B}} \mathrm{x}}$ metallic glasses. The open circles were deduced from critical field data and eqn. (11). The crosses are taken directly from specific heat data.

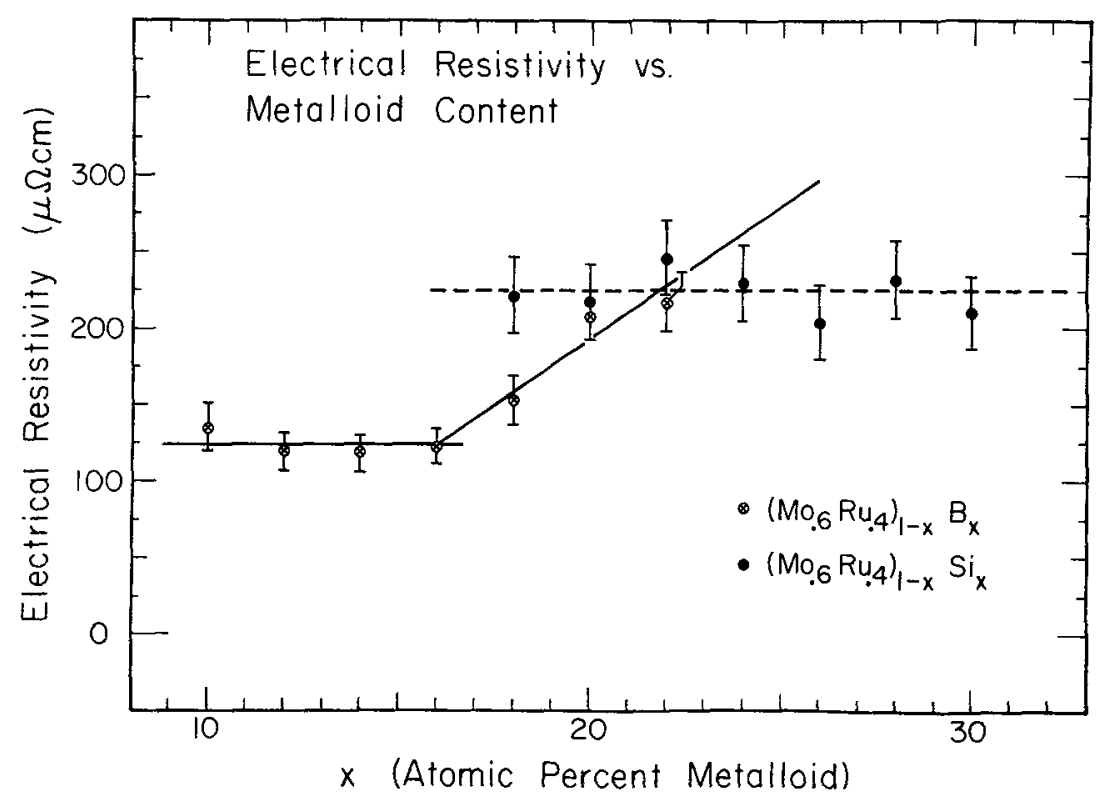

Fig. 8. Electrical resistivity vs. composition for $\left(\mathrm{Mo}_{0.6}{ }^{\mathrm{Ru}} \mathrm{u}_{0.4}\right)_{1-x^{\mathrm{B}} \mathrm{x}}$ and $\left(\mathrm{Mo}_{0} .^{\mathrm{Ru}} 0.4\right)_{1-x^{\mathrm{Si}}}$ metallic glasses. 
role in both the atomic scale structure and electronic properties of metallic glasses. This is best illustrated by the example of $\left(\mathrm{Mo}_{0.6^{\mathrm{Ru}}} 0.4\right)_{1-x^{\mathrm{B}} \times}$ glasses which can be obtained over the composition range $0.10<x<0.24$. Several properties of these alloys have been found to change discontinuously with composition near $x=0.18$. Some examples shown in Figs. 7 and 8 are the average nearest neighbor distance and the electrical resistivity. The former is found to increase with $x$ for $x<0.18$ and decrease for $x>0.18$. The latter is nearly independent for $x$ for $x<0.18$ and increases rapidly for $x>0.18$ (from $v 120 \mu \Omega \mathrm{cm}$ to $250 \mu \Omega \mathrm{cm}$ ). Another example of such behavior is also shown for the metal-metalloid glasses

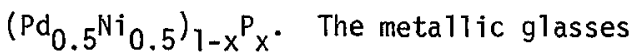
$\left(\mathrm{Mo}_{0.6}{ }^{\mathrm{Ru}} 0.4\right)_{7-\mathrm{x}} \mathrm{Si}_{x}$ are similar but show no discontinuities. The critical field gradient $\left(\mathrm{dH}_{\mathrm{c} 2} / \mathrm{dT}\right)_{\mathrm{T}_{\mathrm{c}}}$ and electrical resistivity can be used together to determine the electronic density of states $D\left(\varepsilon_{F}\right)$ using the relation $(43)$

$$
\left(\frac{\mathrm{dH}_{\mathrm{c} 2}}{\mathrm{dT}}\right)_{T=\mathrm{T}_{\mathrm{C}}}=-4.48 \times 10^{4} \mathrm{Y \rho}\left(0 \mathrm{eK}^{-1}\right)
$$

where $\gamma$ is the equivalent linear coefficient in the electronic heat capacity (in erg $\mathrm{cm}^{-3} \mathrm{~K}^{-2}$ ) and $\dot{p}$ is in units of $(\Omega \mathrm{cm})$. Values of $\gamma$ so obtained are plotted for $\left(\mathrm{Mo}_{0.6} \mathrm{Ru}_{0.4}\right)_{1-x^{\mathrm{B}} \times}$ glasses in Fig. 9 . Specific heat data $(44)$, available for two of the alloys, is included in the figure and agrees extremely well with the $\gamma$ deduced from $\mathrm{H}_{\mathrm{c} 2}$. A precipitious drop in $y$ vs. $x$ occurs near $x=0.18$ with $\gamma \approx 4\left(\mathrm{~mJ} / \mathrm{mole}-K^{2}\right)$ for $x<0.18$ and $y \approx 2\left(\mathrm{mu} / \mathrm{mole}-K^{2}\right)$ for $x>0.18$. We conclude that $D\left(\varepsilon_{F}\right)$ drops by roughly a factor of 2 over this narrow composition interval.

All of the above facts suggest some type phase transition or dramatic structural change near $x=0.18$. The above observations are explained by assuming two different amorphous phases with compositions $x<0.18$ and $x>0.18$. Near $x=0.18$, the alloy would contain both phase in comparable proportions. The technique of small angle $x$-ray scattering SAS was used to obtain evidence of the proposed phase separation ${ }^{(45)}$. Typical small angle scattering spectra are shown in Fig. 10. Curves $A, B, C$, and $D$ show spectra for as quenched foils with $x=0.12,0.14,0.18$, and 0.20 respectively.
A clear feature in the SAS intensity is observed for scattering vector $K \sim 0.3$. This feature is most pronounced for $x=0.18$ an can be interpreted by assuming a phase segregation into domains of characteristic size $\sim 10-15 \AA$ with a typical interdomain distance of $20-30 \AA$.

Results of neutron irradiation studies on $\left(\mathrm{Mo}_{0.6} \mathrm{Ru}_{0.4}\right)_{82^{\mathrm{B}}}{ }_{18}$ have previousiy been reported ${ }^{(46)}$ The SAS spectrum of a neutron irradiated sample is shown by curve $E$. The sample was irradiated to a total fluence of $10^{19} \mathrm{n} / \mathrm{cm}^{2}$ of fast neutrons (1 Mev) over a period of several days. The overall increase in small angle scattering reflects an increase of point defects during irradiation. The large increase in the broad maximum near $K \approx 0.3$ suggests enhanced phase separation. This can be understood since the increased point defect concentration should enhance diffusion at room temperature ${ }^{(47)}$

It is clear that both the electronic structure and atomic scale structure of these metallicglasses are strongly influenced by the phase separation discussed above. On the other hand, the superconducting properties of these materials should reflect only the average properties of the material since the scale of phase separation $10-25 \AA$ is substantially smaller than the coherence length of the superconductor $\xi_{d}(0) \approx 50 \AA$. However, it may be possible to produce a coarser phase separation by thermal aging of the glass. This would result in an intrinsically inhomogeneous superconducting material. An example of clear phase separation on a much larger scale has been observed in a novel family of superconducting metallic glasses $\left(\mathrm{Pb}_{1-y} \mathrm{Sb}_{y}\right)_{1-x^{\mathrm{Au}} \mathrm{x}_{\circ}(48)}$. Here, phase separation on a
scale of $\sim 1000 \AA$ leads to an inhomogeneous superconducting material in which both phases percolate throughout the sample.

\section{SUMMARY}

This article, as mentioned in the introduction, was not intended to be a comprehensive review. The questions raised in the article serve to emphasize that the electronic structure of superconducting metallic glasses is far from being well understood. More generally, the electronic and atomic scale structure of metallic glasses may be considerably more complex than previously recognized. Emphasis has been given to several current problems in the 


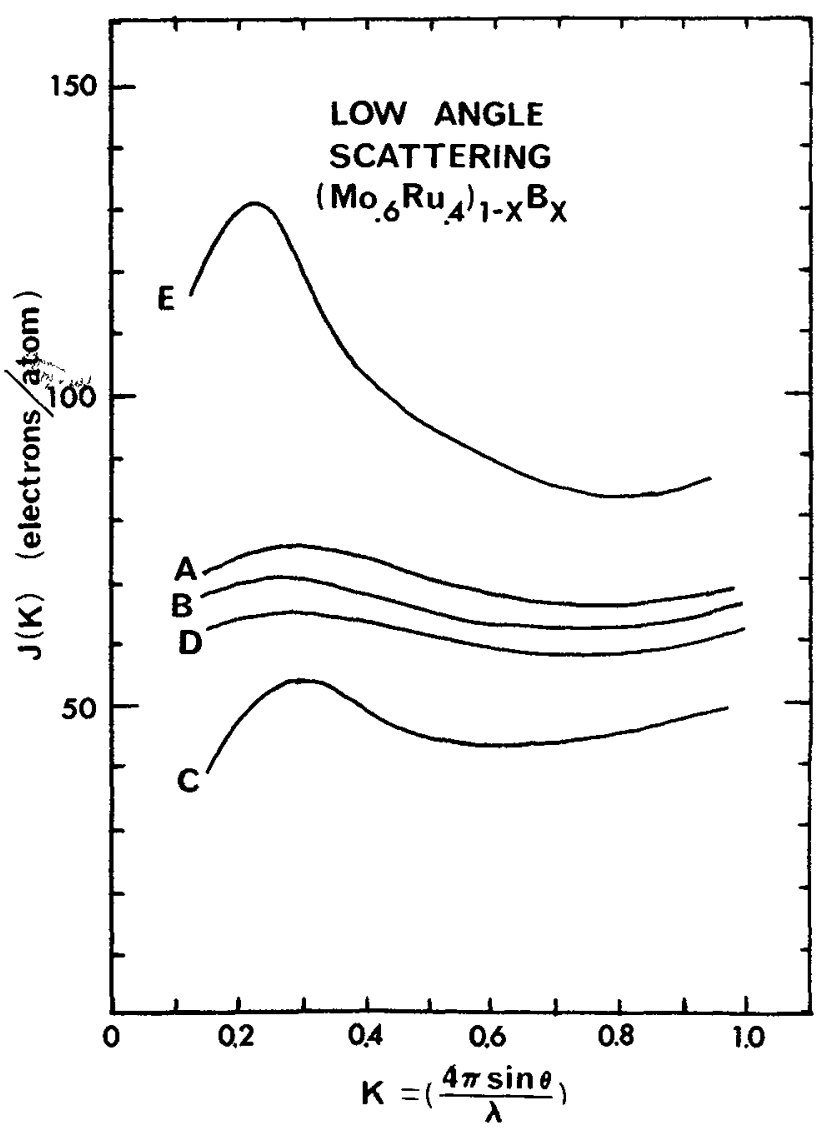

Fig. 10. Small angle scattering intensity, $J(K)$, of $\left(\mathrm{Mo}_{0.6} \mathrm{Ru}_{0.4}\right)_{1-x} \mathrm{~B}_{x}$ alloys. Curves $\mathrm{A}, \mathrm{B}, \mathrm{C}$, and $\mathrm{D}$ are for compositions $x=0.12,0.14,0.18,0.20$ respectively. Curve $E$ is for a neutron irradiated specimen with $x=0.18$. A total fluence of $10^{19} \mathrm{n} / \mathrm{cm}^{2}$ of fast (1 MeV) neutrons was accumulated over a period of 2 days.

study of amorphous superconductors. Hopefully, future research will provide a deeper insight into the nature of these novel materials.

\section{REFERENCES}

1. H. Buckel and R. Hilsch, Z. Phys, , 138, 109 (1954).

2. W. Buckel and R. Hilsch, Z. Phys., 146, 27 (1956).

3. G. Bergmann, Phys. Reports, 27C, 161 (1976).

4. W. L. Johnson, Glassy Metals, ed. by H. Guntherodt and $H$. Beck (Springer Verlag, Heidelberg, West Germany, 1980).

5. W. L. Johnson, S. J. Poon, and P. Duwez, Phys. Rev. B, 11, 150 (1975).

6. K. Togano and K. Tachikawa, Phys. Lett., 54A, 205 (1975)

7. H. L. Johnson, Proc. III Int. Conf. on Rapidiy Quenched Metals, ed. by B. Cantor, (The British Metals Society, Chameleon Press, London, 1978) Vol. II p. 1.

8. P. Duwez, Progress in Solid State Chemistry, (Pergamon, 0xford, 1966) Vol. 3.

9. H. L. Mcilitlan, Phys. Rev., 167, 331 (1968).

10. J. W. Garland, as quoted in T. T. Chen, J. T. Chen, J. D. Les]ie, and H. J. Smith, Phys. Rev. Lett., 22, 526 (1969).

11. J. II. Ziman, Phi1. Mag., 6, 1013 (1961); C. C. Bradley, T. E. Faber, E. G. Wilson, and J. M. Ziman, Phi1. Mag., 7865 (1962).

12. H. Guntherodt, Metallic Glasses, (American Society for Metals, Metals Park, Ohio, 1978) p. 247-272.

13. J. H. Ziman, Principles of the Theory of Solids (Cambridge University Press, England, 1972) Chap. 7.

14. G. Bergmann and D. Rainer, Z. Phys., 263, 59 (1973).

15. K. Knorr and N. Barth, Solid State Comm., $\underline{8}$, 1085 (1970).

16. J. E. Jackson, C. V. Briscoe, H. Wuht, Physica, 55, 447 (1971).

17. J. Friedel, Physics of lletals, ed. by J. M. Ziman, (Cambridge Press, Cambridge, England, 1969) p. 340-403.

18. F. Cyrot Lackmann, Adv. in Phys., 16393 (1967).

19. F. Cyrot Lackmann, J. Phys. 35, C4-109 (1974).

20. C. M. Varma, R. C. Dynes, Superconductivity in d- and f-band Metals, ed. by D. H. Douglass (Plenum Press, New York, 1976).

21. W. L. Johnson, S. J. Poon, J. Durand, and P. Duwez, Phys. Rev. B18, 206 (1978). 
22. A. Amamou and W. L. Johnson, (to be published in Solid State Comm.).

23. B. Schroeder, W. Grobman, W. L. Johnson, C. C. Tsuei, and P. Chaudhari, Solid State Comm. 28, 631 (1978).

24. L. F. Mattheiss, Phys. Rev. B1, 373, (1970): Phys. Rev., 151, 450 (1966).

25. J. Friedel, Adv. Phys., 3,446 (1954).

26. F. Heiniger, E. Bucher, and J. Muller, Phys. Kond. Mat., 5, 243 (1966); F. J. Morin and J. I. Maita, Phys. Rev. 129, 1115 (1963).

27. N. F. Mott and T. Davis, Electronic Processes in Non-Crystalline Materials (Clarendon Press, Oxford, 1979) Chaps. 2,5.

28. P. W. Anderson, Phys. Rev. 109, 1492 (1958).

29. D. Saint-James, G. Sarma, and E. J. Thomas, Type II Superconductivity (Pergamon Press, Oxford, London, 1969) Chaps. 2,5.

30. E. R. Domb and W. L. Johnson, J. Low Temp. Phys., 33, 29 (1978).

31. H. Tenhover, W. L. Johnson, and C. C. Tsuei, (to be published, Phys. Rev. Lett., 1980).

32. W. L. Johnson and C. C. Tsuei, Phys. Rev. B, 13, 4827 (1976).

33. W. L. Johnson, C. C. Tsuei and P. Chaudhari, Phys. Rev. B, 17, 2884 (1978).

34. K. Maki, Physics, 1, 127 (1964).

35. N. R. Werthamer, E. Hel fand, and P. C. Hohenberg, Phys. Rev., 147, 295 (1966).

36. A. M. Clogston, Phys. Rev. Lett., 9, 266 (1962).

37. D. Rainer, G. Bergmann, and V. Eckhardt, Phys. Rev. B, 85324 (1973).

38. No anisotropy effects should be expected in amorphous metals since no preferred directions exist.

39. R. Meservey, P. M. Tedrow, Phys. Rev. Lett., 43, 384 (1979).

40. S. Barasic, J. Labbe, and J. Friede1, Phys. Rev. Lett., 25, 919 (1970).

41. A. R. Williams and W. L. Johnson, J. Non-Cryst. Solids, 34, 121 (1979).

42. W. L. Johnson and A. R. Withiams, Phys. Rev. B, 20, 1 (1979).

43. T. P. Orlando, E. J. Hicliff Jr., S. Foner, and II. R. Beasley, Phys. Rev. B, 19, 4545 (1979).

44. S. Hopkins and W. L. Johnson, unpublished results.
45. See for example A. Guinier, Small Angle Scattering of X-rays (John Wiley, New York, 1955)

46. A. Kramer, H. L. Johnson, and C. Cline, Applied Phys. Lett., 35,815 (1979).

47. See for example R. S. Nelson in Radiation Bamage Processes in Materials, ed. by C. H. S: Dupuy (Noordhoff, Legden, 1975) p. 261.

48. C. 0. Kim and W. L. Johnson, to be published in Phys. Rev. B (1980). 